\title{
A Machine Learning Approach Applied to Gynecological Ultrasound to Predict Progression- Free Survival in Ovarian Cancer Patients
}

\section{Francesca Arezzo \\ Gennaro Cormio \\ Daniele La Forgia \\ Michele Mongelli \\ Claudio Lombardi \\ Gerardo Cazzato \\ Ettore Cicinelli \\ Vera Loizzi}

https://orcid.org/0000-0002-8914-9594

Carla Mariaflavia Santarsiero

\section{Research Article}

Keywords: Machine learning, ovarian cancer, gynecological ultrasound, progression-free survival

Posted Date: February 28th, 2022

DOI: https://doi.org/10.21203/rs.3.rs-1382403/v1

License: (c) (i) This work is licensed under a Creative Commons Attribution 4.0 International License.

Read Full License 


\section{Abstract}

In a growing number of social and clinical scenarios, machine learning $(\mathrm{ML})$ is emerging as a promising tool for implementing complex multi parametric decision-making algorithms. Regarding ovarian cancer (OC), despite the standardization of features that can support the discrimination of ovarian masses into benign and malignant, there is a lack of accurate predictive modeling based on ultrasound (US) examination for progression-free survival (PFS). This retro-spective observational study analysed patients with epithelial ovarian cancer (EOC) who were followed in a tertiary centre from 2018 to 2019.

Demographic features, clinical characteristics, information about the surgery and post-surgery histopathology were collected. Additionally, we recorded data about US examinations according to the International Ovarian Tumor Analysis (IOTA) classification. Our study aimed to realise a tool to predict 12month PFS in patients with OC based on a ML algorithm applied to gynecological ultrasound assessment. Proper feature selection was used to determine an attribute core set. Three different machine learning algorithms, namely Logistic Regression (LR), Random Forest (RFF), and K-nearest neighbors (KNN), were then trained and validated with five-fold cross-validation to predict 12-month PFS. Our analysis included n. 64 patients. The attribute core set used to train machine learning algorithms included age, menopause, CA125 value, histotype, FIGO stage and US characteristics such as major lesion diameter, side, echogenicity, color score, major solid component diameter, presence of carcinosis. RFF showed the best performance (accuracy 93.7\%, precision $90 \%$, recall $90 \%$, area under receiver operating characteristic curve (AUROC) 0.92). We developed an accurate ML model to predict 12month PFS.

\section{Introduction}

\subsection{Ovarian cancer}

Ovarian cancer $(\mathrm{OC})$ is the seventh-most-diagnosed cancer among women worldwide and the secondmost-common gynecological malignancy. It represents appromixmately 14,000 deaths in 2020 in the US [1].

Up to $90 \%$ of ovarian cancers are epithelial ovarian cancer (EOC) types. OC has multiple cellular origins [2]. The term tubo-ovarian cancer is often used because $\mathrm{OC}$ can arise as an ovarian or fallopian-tube mass or primary peritoneal cancer [3].

Type I tumors (low-grade serous, mucinous, endometrioid, and clear cell) occurring in the ovary are less aggressive and are therefore more easly diagnosed at an early stage because they tend to grow slowly. Type II tumors (high-grade serous carcinomas (HGSC), undiffer-entiated carcinomas, and carcinosarcomas) may originate from the tubal and/or ovarian surface epithelium, and are more aggressive [4-6]. 
The absence of proper screening and diagnostic procedures to detect $\mathrm{OC}$ at an early stage as well as the rapid spread of disease through the peritoneal surface are leading factors in the $\mathrm{OC}$ lethality $[7,8]$. Nowadays, there is a lack of an accurate protocol to identify high-risk patients.

Therefore, identifying tools for accurate screening and early diagnosis and prognosis of $\mathrm{OC}$ represents a currently unmet clinical need.

In addition, the role of Ultrasound (US) in OC is evolving. US is a cheap, non-invasive and well-recognized image modality for diagnosis and evaluation of OC [9].

The International Ovarian Tumor Analysis (IOTA) group established a standardized lexicon that includes all appropriate descriptors and definitions of the sonographic appearance characteristic of normal ovaries and ovarian lesions. In order to simplify the sonographer's assessment in differentiating benign from malignant adnexal masses, they also developed the Simple Rules classification system and the Assessment of Different Neoplasia in the Adnexa (ADNEX) model [10-16]. The Society of Radiologists in Ultrasound consensus statement $[17,18]$ and the Gynecologic Imaging Reporting and Data System, also known as GI-RADS [19], are other proposed systems for the characterization and management of ovarian masses (OM) [20].

In 2018, the Ovarian-Adnexal Reporting and Data System (O-RADS) created a risk stratification classification for consistent follow up and management in clinical practice [21].

But quickly, a simple description of the tumor and of its extension may not be sufficient. The application of precision medicine could help answering a question about early response to treatment, best timing for surgery, prognosis or molecularly-targeted drug.

\subsection{Machine Learning}

In a growing number of social and clinical scenarios, machine learning $(\mathrm{ML})$ is emerging as a promising tool for the implementation of complex multi-parametric decision-making algorithms [22,23]. In that sense, a ML approach is a potential gamechanger [24]. In fact, in addition to detecting linear patterns in analyzed data, it can unravel complex non-linear relationships between patient attributes that cannot be solved by traditional statistical methods, merging them to produce a prediction or a probability for a given outcome $[22,25,26]$.

ML is a step towards precision medicine, leading to improved patient profiling and personalised treatment. Supervised $\mathrm{ML}$ algorithms have been shown to be effective in predicting treatment responses and disease progression in patients affected with heterogeneous diseases [27, 28].

Regarding OC, despite the standardization of features that can support the discrimination of ovarian masses into benign and malignant, there is the lack of accurate predictive modeling based on US examination for PFS. 


\section{Materials And Methods}

In this retrospective observational study, we analyzed consecutive patients with EOC who were followed in a tertiary center from 2018 to 2019.

Demographic features (age), clinical characteristics (parity, menopause, CA125 value, BRCA mutation, treatment) were collected as well as information about surgery (surgical procedures, residual tumor) and post-surgery histopathology (histotypes, grading, FIGO stage). Additionally, we recorded data about transvaginal and/or transabdominal US examinations according to IOTA classification (unilateral lesion, side, largest diameter of lesion, type of tumor, echogenicity of cyst fluid in tumors, color score, diameter of largest solid component, shadows, ascites, carcinosis, subjective assessment).

Our study aimed to realized a tool to predict 12-month PFS in patients with OC based on a ML algorithm applied to gynecological ultrasound assessment.

In total, the original database included n. 64 patients and $n .23$ variables.

Appropriate feature selection was used to determine an attribute core set (see Supplementary Materials for further details).

This study followed STARD guidelines [29] and the TRIPOD statement [30].

The ML algorithms were aimed at forecasting PFS at 12-month follow up.

Student's t-test for paired samples or Wilcoxon matched-pair signed-rank test were used as appropriate to identify difference between continuous variables between different observation periods. McNemar's test was used to identify the difference among dummy variables between

The attribute core set used to train the algorithms was determined using a recursive feature elimination (RFE) wrapper based on a decision tree algorithm with extreme gradient boosting (XGBoost) [31]; in brief, this algorithm automatically selects from all the recorded attributes (n. 23) the best number of features on their importance for the given outcome predictions (PFS at 12 months). Feature selection can counteract overfitting problems and improve classification performance. RFE elimination method is one of the commonly used feature selection methods for small samples problems [32,34] (For further details about RFE see Supplementary Materials).

The entire analysis was implemented in a Python 3.6 environment using scikit-learn (ver.0.22.1) and XGBoost (ver. 1.1.0) libraries [31,35]. After Z-score normalization, we performed a Bayesian ridge conditional ridge imputation [36] for missing data. The latter method proved to be the most accurate method of imputation for obstetrics and gynecology datasets [37] (see Supplementary Materials for further details).

Three different classifiers, both linear and non-linear, were trained and cross-validated with five-fold crossvalidation using the core set of attributes recovered from the RFE to predict 12-month PFS. 
While logistic regression (LR) was almost always the algorithm of choice to find independent predictors in multivariate models, it must be noticed that the study hypotheses were usually based on the unrealistic assumption that the association between the prognostic factors and clinical outcomes is direct and isolated. In contrast, LR is not suitable for the modeling of non-independent variables. For this reason, along with usual LR, for linear modeling we used the non-parametric K-nearest neighbors (KNN) and random forest (RFF)[36] algorithms. The latter models have recently been shown to accurately predict important outcomes for woman's health, even in the presence of non-linear patterns in data [38-40]. Furthermore, we choose RFF because there is evidence of accurate performance in case of unbalanced data, which is often the case of clinical datasets [41]. We also ran RFF using cost-sensitive training (using the argument class weight = "balanced" in scikit-learn) to try to overcome unbalanced class issue.

A repeated grid-search with cross-validation was used for optimal hyperparameter tuning to maximize the classifiers' performance [42] (See Supplementary Material for hyperparameter fine-tuning).

For each classifier, we plotted ROC curves, and then area under receiver operating characteristic curve (AUROC) was determined.

Then, based on the optimal probability cut-off (Youden's Index)[43] classifiers' performance was compared with the following metrics:

- Accuracy $=\frac{\text { truepositives }+ \text { truenegatives }}{\text { truepositives }+ \text { truenegatives }+ \text { falsepositives }+ \text { falsenegatives }}$

- Recall (True Positive Rate $(T P R))=\frac{\text { truepositives }}{\text { truepositives + falsenegatives }}$,

- Precision $=\frac{\text { truepositives }}{\text { truepositives }+ \text { falsepositives }}$.

In general, a classification model forecasts a binary outcome for a given observation and class. In the process of predicting, a model may output the probability of an observation belonging to each possible class. This case allows some flexibility in the way predictions are interpreted and presented, allowing the choice of a threshold, such as the aformentioned Youden's index [44].

For a model to be reliable, the estimated class probabilities should reflect the true underlying probability of the sample. To check these assumptions, a diagnostic calibration curve for the candidate best classifier was also plotted [44].

The study was conducted in accordance with the Declaration of Helsinki, and the protocol was approved by the Scientific Board University of Bari, Bari, Italy. All patients had signed a consent to use the data in scientific purposes.

\section{Results}

Our analysis included n. 64 patients with diagnosis of EOC. Demographic and clinical characteristics, information about surgery procedures, post-surgery histopathology and US features are outlined in Table 
Page $6 / 22$ 
Table 1

Cohort characteristics.

\begin{tabular}{|c|c|}
\hline Age at diagnosis (years), mean \pm SD & $54,1 \pm 14,9$ years \\
\hline Parity, median (IQR) & $1(0-2)$ \\
\hline Menopause, n. (\%) & $28 / 64(43.7 \%)$ \\
\hline CA125 $(\mathrm{U} / \mathrm{mL})$, mean \pm SD & $828,25( \pm 2018.82)$ \\
\hline BRCA1m, n. (\%) & $4 / 64(6.25 \%)$ \\
\hline BRCA2m, n. (\%) & $4 / 64(6.25 \%)$ \\
\hline BRIP1m, n. (\%) & $2 / 64(3.12 \%)$ \\
\hline Unilateral tumor, n. (\%) & $34 / 64(53.1 \%)$ \\
\hline \multicolumn{2}{|l|}{ Side, n. (\%) } \\
\hline Right & $16 / 64(25 \%)$ \\
\hline Left & $18 / 64(28.1 \%)$ \\
\hline Middle & $30 / 64(46.9 \%)$ \\
\hline Largest diameter of lesion $(\mathrm{mm})$, mean $\pm \mathrm{SD}$ & $113.6 \pm 57.6$ \\
\hline \multicolumn{2}{|l|}{ Type of tumor, n. (\%) } \\
\hline Unilocular & 0 \\
\hline Unilocular-solid & $8 / 64(12.5 \%)$ \\
\hline Multilocular & $2 / 64(3.1 \%)$ \\
\hline Multilocular-solid & $28 / 64(43.7 \%)$ \\
\hline Solid & $26 / 64(40.7 \%)$ \\
\hline \multicolumn{2}{|c|}{ Echogenicity of cyst fluid in tumors not classified as solid, n. (\%) } \\
\hline Anechoic & $22 / 64(34.4 \%)$ \\
\hline Ground glass & $6 / 64(9.4 \%)$ \\
\hline Low level & 10/64 (15.6\%) \\
\hline \multicolumn{2}{|l|}{ Color Score, n. (\%) } \\
\hline 1 & $8 / 64(12.5 \%)$ \\
\hline 2 & $6 / 64(9.4 \%)$ \\
\hline 3 & $18 / 64(28.1 \%)$ \\
\hline 4 & $32 / 64(50 \%)$ \\
\hline
\end{tabular}




\begin{tabular}{|c|c|}
\hline Age at diagnosis (years), mean \pm SD & $54,1 \pm 14,9$ years \\
\hline Diameter of largest solid component $(\mathrm{mm})$, mean \pm SD & $71.1 \pm 45.1$ \\
\hline Shadows, n. (\%) & $8 / 64(12.5 \%)$ \\
\hline Ascites, n. (\%) & $18 / 64(28.1 \%)$ \\
\hline Carcinosis, n. (\%) & $20 / 64(31.2 \%)$ \\
\hline \multicolumn{2}{|l|}{ Diagnosis on basis of subjective assessment, n. (\%) } \\
\hline Benign & $8 / 64(12.5 \%)$ \\
\hline Malignant & $56 / 64(87.5 \%)$ \\
\hline \multicolumn{2}{|l|}{ Surgery, n. (\%) } \\
\hline Open surgery & $49 / 64(76.5 \%)$ \\
\hline Laparoscopy & $15 / 64(23.5 \%)$ \\
\hline \multicolumn{2}{|l|}{ Residual Tumor, n. (\%) } \\
\hline RO & $48 / 64(75 \%)$ \\
\hline $\mathrm{R} 1$ or $\mathrm{R} 2$ & $16 / 64(25 \%)$ \\
\hline \multicolumn{2}{|l|}{ Histotypes, n. (\%) } \\
\hline High-grade serous & $42 / 64(65.6 \%)$ \\
\hline Endometrioid & $10 / 64(15.6 \%)$ \\
\hline Clear cell & $8 / 64(12.5 \%)$ \\
\hline Mucinous & $4 / 64(6.3 \%)$ \\
\hline \multicolumn{2}{|l|}{ Grading, n. (\%) } \\
\hline G1 & $12 / 64(18.7 \%)$ \\
\hline $\mathrm{G} 2$ & $2 / 64(6.3 \%)$ \\
\hline G3 & $48 / 64(75 \%)$ \\
\hline \multicolumn{2}{|l|}{ FIGO Stage, n. (\%) } \\
\hline I & $22 / 64(34.4 \%)$ \\
\hline II & $2 / 64(3.1 \%)$ \\
\hline III & $26 / 64(40.6 \%)$ \\
\hline IV & $14 / 64(21.9 \%)$ \\
\hline Treatment, n. (\%) & \\
\hline
\end{tabular}




\begin{tabular}{|ll|}
\hline Age at diagnosis (years), mean $\mathbf{\text { SD }}$ & $\mathbf{5 4 , 1} \mathbf{\pm 1 4 , 9}$ years \\
\hline No treatment & $6 / 649.4 \%)$ \\
\hline Neoadjuvant therapy & $24 / 64(37.5 \%)$ \\
\hline Adjuvant chemotherapy & \\
\hline Paclitaxel-Carboplatin & $24 / 64(37.5 \%)$ \\
\hline Paclitaxel-Carboplatin-Bevacizumab & $2 / 64(3.1 \%)$ \\
\hline Paclitaxel-Carboplatin-Parp inhibitor & $8 / 64(12.5 \%)$ \\
\hline
\end{tabular}

Patients had a mean age ( \pm SD) of $54,1 \pm 14,9$ years at diagnosis and n. 28/64 (43.7\%) were menopausal patients. CA 125 median value was $828,25( \pm 2018.82) \mathrm{U} / \mathrm{mL}$. Four out of $64(6.25 \%)$ women had BRCA1 mutation, n. 4/64 (6.25\%) women had BRCA2 mutation and n. 2/64 (3.12\%) women had BRIP1 mutation.

Concerning US characteristics, n. 34/64 (53.1\%) patients had a unilateral mass and the median greatest diameter was $113.6 \pm 57.6 \mathrm{~mm}$. The most common tumor type was multilocular-solid (28/64 (43.7\%)), followed by solid (26/64 (40.7\%)), unilocular-solid (8/64 (12.5\%)), and multilocular (2/64 (3.1\%)) masses. The median diameter of the largest solid component was $71.1 \pm 45.1 \mathrm{~mm}$. The most common echogenicity of cyst fluid was anechoic (22/64 (34.4\%)), followed by low level echogenicity in n.10/64 (15.6\%) and ground glass echogenicity in n. 6/64 (9.4\%). Most of these tumors showed intense vascularity on color Doppler examination (32/64 (50\%)) and n. 18/64 (28.1\%) moderate vascularity. Based on the subjective assessment by the original US examiner, n. 56/64 (87.5\%) masses were classified as malignant and n. $8 / 64(12.5 \%)$ as benign tumors. Ultrasonographic evaluation revealed ascites in n. 18/64 (28.1\%) and carcinosis in n. 20/64 (31.2\%). Only n. 8/64 (12.5\%) revealed shadows.

Concerning the surgical procedure, n. 49/64 (76.5\%) underwent open surgery and n. 15/64 (23.5\%) underwent laparoscopy. Forty eight out of 64 (75\%) presented no residual tumor; n. 16/64 (25\%) presented microscopic (R1) or macroscopic (R2) residual tumor.

On histopathological analysis, histotypes were n. 42/64 (65.6\%) high-grade serous carcinoma, n. 10/64 (15.6\%) endometrioid, n. 8/64 (12.5\%) clear cell and 4/64 (6.3\%) mucinous. Grade was G1 in n. 12/64 (18.7\%), G2 in n. 2/64 (6.3\%), G3 in n. 48/64 (75\%). Most tumors were FIGO Stage III (26/64 (40.6\%)), followed by FIGO stage I (22/64 (34.4\%)) and FIGO stage IV (14/64 (21.9\%)). Only n. 2/64 (3.1\%) had a FIGO stage II.

Twenty four out of 64 (37.5\%) were treated with neoadjuvant chemotherapy with paclitaxel-carboplatin, $\mathrm{n}$. 24/64 (37.5\%) with adjuvant chemotherapy with paclitaxel-carboplatin, n. 8/64 (12.5\%) adjuvant chemotherapy with paclitaxel-carboplatin and parp inhibitor and n. 2/64 (3.1\%) paclitaxel-carboplatin and bevacizumab. Six out of $64(9.4 \%)$ required no treatment. 12-month PFS was achieved by $46 / 64$ patients (71.9\%, unbalanced classes). 
As detailed in Fig. 1, RFE retrieved an attribute core set used to train machine learning algorithms including age, menopause, CA125 value, histotype, FIGO stage and US characteristics such as major lesion diameter (Fig. 2), side, echogenicity (Fig. 3), color score (Fig. 4), major solid component diameter (Fig. 5), presence of carcinosis (Fig. 6).

The attribute core set used to train machine learning algorithms is reported in Fig. 1. RFF showed an accuracy of 0.93 , AUROC 0.92 .

The final dataset had a dimensionality of 64 columns $\times 12$ rows ( $\mathrm{n} .11$ selected attributes plus $\mathrm{n} .1$ target class (PFS at 12 months, as above mentioned).

As reported in Table 2, at optimal cut-off (Youden's index), RFF (n. estimators $=500$, depth $=5$ ) showed the best performance (accuracy $93.7 \%$, precision 90\%, TPR 90\%, AUROC 0.92), outperforming LR (accuracy $82 \%$, precision $80.1 \%$, TPR $84.1 \%$, AUROC 0.81 ), and KNN (n. of neighbors = 5) (accuracy $73.6 \%$, precision $76.5 \%$, TPR $83.3 \%$, AUROC 0.69).

Table 2

Algorithms Performance. Algorithm with the best performance on five-fold cross validation is indicated in bold. Accuracy, Recall, Precision and AUROC for RFF, were significantly better than other algorithms' ones.

\begin{tabular}{|llllll|}
\hline & Youden's Index Cut-Off & Accuracy (\%) & TPR (\%) & Precision (\%) & AUROC \\
\hline LR & 0.64 & 82 & 84.1 & 80.1 & 0.81 \\
\hline RFF & 0.77 & 93.7 & 90 & 90 & 0.92 \\
\hline KNN & 0.68 & 73.6 & 83.3 & 76.5 & 0.69 \\
\hline
\end{tabular}

AUROC: Area under receiver operating characteristics curve; LR: Logistic Regression; KNN: K-nearest neighbors; RFF: Random Forest; TPR: True Positive Rate.

In Fig. 7, ROC curve for RFF (box A), LR (box B) and KNN (box C) models was reported.

In Fig.8 calibration diagnostic has been plotted for RFF; PFS roughly happened with an observed relative frequency consistent with the forecast value, showing an acceptable calibration curve. We would expect the match between predicted frequencies and observed frequencies to increase with a larger dataset.

\section{Discussion}

The keystone of survival analyses in cancer research has historically been Cox proportional hazard regression model, being a surrogate for estimating treatment efficacy and safety. This model is based on the assumption of linear association. However, many clinicopathologic features show a non-linear association in medicine [45].

The ML approach has recently brought an unprecedented growth of applications to medical imaging. 
In the study of OC, since 1999, artificial neural networks [46,47] have been applied to classify US image into benign and malignant, but image features were manually measured and provided by the investigators.

In 2015, Kazendar et al. [48] developed a fully automatic ML classifier stratifying US images as benign or malignant masses with an accuracy of $77 \%$ when images were enhanced with a Local Binary Pattern operator.

Recently, due to the wide availability of digital medical images and the technical advances in hardware and software, ML has also been applied in conjunction with radiomic analysis.

In a study by Chiappa et al. [49], ML and radiomics were applied to transvaginal ultrasonography (TUS) to implement a decision support system (DSS) for predicting the risk level of malignancy of OM.

The DSS was based on a set of three radiomic ML models, named as solid masses, cystic masses and mixed masses. These radiomic models were integrated with information about presence/absence of acoustic shadows and serum CA 125 level, considering two different thresholds according to menopausal status.

This addition integrate the malignancy risk predicted by each of the three TUS radiomic models.

The DSS was based on TUS imaging and serum CA125 level and showed 91\% accuracy, 100\% sensitivity, and $80 \%$ specificity in independent tests.

Martinez-Mas et al. [50] realised a ML algorithms aimed to perform the automatic categorization of OC from US images. They analyzed 348 images. For each patient case and US image, its input features were previously extracted using Fourier descriptors calculated over the Region Of Interest (ROI). Then, four ML algorithms were considered to perform the classification stage: KNN, Linear Discriminant (LD), Support Vector Machine (SVM) and Extreme Learning Machine (ELM). LD, SVM and ELM reported more than 85\% accuracy.

Regarding ML applications in the clinical management of OC patients, Hwangbo et al. [51] aimed to develop ML models predicting platinum sensitivity in patients with HGSC. Using the stepwise selection method, based on the AUC values, six variables associated with platinum sensitivity were selected: age, initial serum CA 125 levels, neoadjuvant chemotherapy, pelvic lymph node status, pelvic tissue involvement other than uterus and tubes, and small bowel and mesentery involvement. Based on these variables, predictive models were constructed using four ML algorithms, LR, RFF, SVM and deep neural network. Evaluation of model performance using the five-fold cross-validation method identified the LRbased model as the best for identification platinum-resistant cases. Therefore they developed a webbased nomogram adapting the LR model results for clinical utility.

Also attempting to improve treatment choices of OC patients, Shannon et al. [52] developed a ML tool to identify predictive molecular markers for cisplatin chemosensitivity. 
CYTH3, GALNT3, S100A14, and ERI1 were the four potential biomarkers identified. Validation was performed on a cohort of $\mathrm{n} .50$ patients who underwent surgery followed by adjuvant carboplatin. Predictive models were established to predict chemosensitivity. The four biomarkers were also evaluated for their ability to prognosticate overall survival (OS) in three OC microarray expression datasets from The Gene Expression Omnibus. The extreme gradient boosting (XGBoost) algorithm was selected for the final model to validate the accuracy in an independent validation dataset $(n=10)$. CYTH3 and S100A14, followed by nodal stage, were the most important features. The signature of the four genes had a comparable prognosis to clinical information for two-year survival.

To date, only few studies attempted to apply ML to ultrasound evaluation of adnexal masses in order to predict benign or malignant histology.

On the other hand, some authors applied ML using only clinical and laboratory data to predict treatment response. To our best knowledge, this is the first $M L$ algorithm basing on clinical, surgical, histophalogical and US features in order to predict PFS in patients diagnosed with OC.

The main limitation of our study is the low sample size, which in fundamental in ML research. Neverdless RFF as proven robust in previous studies with low or similar sample size [23]. In order to be adopted in clinical practice, the algorithm will need extensive external validation on larger prospective cohorts.

In gynecologic oncology, ML is a step towards precision medicine, leading to an improved patient profile and personalised treatment.

This model could be applied at the time of diagnosis in order to predict 12-month PFS in patients with OC. This algorithm was applied to gynecological US evaluation, which requires few easy-to-collect attributes. Further studies are needed to assess the potential of ML algorithms in routine gynecologic care.

\section{Declarations}

Acknowledgments: We thank the association "ACTO - alleanza contro il tumore ovarico" for supporting the research activity of Francesca Arezzo with the "Adele Leone" grant.

Funding: This research received no external funding.

Competing Interests: The authors have no relevant financial or non-financial interests to disclose.

Author Contributions: Francesca Arezzo and Vera Loizzi performed the study concepyion. Gennaro Cormio, Claudio Lombardi and Daniele La Forgia contributed to the study design. Material preparation and data collection were performed by Carla Mariaflavia Santarsiero and Michele Mongelli. The first draft of the manuscript was written by Francesca Arezzo and Gennaro Cormio. Claudio Lombardi, Erika Silvestris and Daniele La Forgia performed the data visualization. The manuscript was reviewed by Francesca Arezzo, Michele Mongelli and Carla Mariaflavia Santarsiero under the supervision of Gennaro 
Cormio. The project was administrated by Vera Loizzi, Ettore Cicinelli and Francesca Arezzo. All authors read and approved the final manuscript.

Ethics approval: The study was conducted according to the guidelines of the Declaration of Helsinki, and approved by the Ethics Committee of Azienda Ospedaliera Policlinico Consorziale - University of Bari, IT (protocol code 6398, date of approval 10.06.2020)."

Consent to participate: Informed consent was obtained from all subjects involved in the study at baseline consultation.

Consent to publish: The authors affirm that human research participants provided informed consent for publication of the images in Figures 2, 3, 4, 5 and 6.

Data Availability Statement: Data are not freely available due to local Ethics Committee privacy issues. Authors will consider data sharing upon specific request to local Ethics Committee.

\section{Author Contributions:}

Arezzo Francesca: conceptualization , resources, manuscript writing, manuscript editing, project administration

Cazzato Gerardo: resources

Cicinelli Ettore: project administration

Cormio Gennaro: methodology, manuscript writing, supervision

La Forgia Daniele: methodology, visualization

Loizzi Vera: conceptualization, project administration

Lombardi Claudio: methodology, visualization

Mongelli Michele: manuscript editing, data curation

Santarsiero Carla Mariaflavia: manuscript editing, data curation

\section{References}

1. Siegel RL, Miller KD, Jemal A. Cancer statistics, 2020. CA Cancer J Clin. 2020;70(1):7-30.

2. Loizzi V, Cormio G, Resta L, Rossi CA, Di Gilio AR, Cuccovillo A, et al. Neoadjuvant chemotherapy in advanced ovarian cancer: a case-control study. Int J Gynecol Cancer. 2005;15(2):217-23.

3. Loizzi V, Leone L, Camporeale A, Resta L, Selvaggi L, Cicinelli E, et al. Neoadjuvant Chemotherapy in Advanced Ovarian Cancer: A Single-Institution Experience and a Review of the Literature. Oncology. 
2016;91(4):211-6.

4. Loizzi V, Selvaggi L, Leone L, Latorre D, Scardigno D, Magazzino F, et al. Borderline epithelial tumors of the ovary: Experience of 55 patients. Oncol Lett. 2015;9(2):912-4.

5. Forstner R. Early detection of ovarian cancer. Eur Radiol. 2020;30(10):5370-3.

6. Cazzato G, Colagrande A, Arezzo F, Resta L, Ingravallo G. "Black Ovaries": An Uncommon Case of First Systemic Recurrence of Melanoma. Reports. 2021;4(2):13.

7. Cormio G, Loizzi V, Carriero C, Putignano G, Selvaggi L. Spleen involvement in women with ovarian cancer. Eur J Gynaecol Oncol. 2009;30(4):384-6.

8. Arezzo F, Cazzato G, Loizzi V, Ingravallo G, Resta L, Cormio G. Peritoneal Tuberculosis Mimicking Ovarian Cancer: Gynecologic Ultrasound Evaluation with Histopathological Confirmation. Gastroenterology Insights. 2021;12(2):278-82.

9. Arezzo F, Loizzi V, La Forgia D, Abdulwakil Kawosha A, Silvestris E, Cataldo V, et al. The Role of Ultrasound Guided Sampling Procedures in the Diagnosis of Pelvic Masses: A Narrative Review of the Literature. Diagnostics. 2021;11(12):2204.

10. Patel-Lippmann KK, Sadowski EA, Robbins JB, Paroder V, Barroilhet L, Maddox E, et al. Comparison of International Ovarian Tumor Analysis Simple Rules to Society of Radiologists in Ultrasound Guidelines for Detection of Malignancy in Adnexal Cysts. AJR Am J Roentgenol. 2020;214(3):694700 .

11. Abramowicz JS, Timmerman D. Ovarian mass-differentiating benign from malignant: the value of the International Ovarian Tumor Analysis ultrasound rules. Am J Obstet Gynecol. 2017;217(6):65260.

12. Timmerman D, Van Calster B, Testa A, Savelli L, Fischerova D, Froyman W, et al. Predicting the risk of malignancy in adnexal masses based on the Simple Rules from the International Ovarian Tumor Analysis group. Am J Obstet Gynecol. 2016;214(4):424-37.

13. Dakhly DMR, Gaafar HM, Sediek MM, Ibrahim MF, Momtaz M. Diagnostic value of the International Ovarian Tumor Analysis (IOTA) simple rules versus pattern recognition to differentiate between malignant and benign ovarian masses. Int J Gynaecol Obstet. 2019;147(3):344-9.

14. Timmerman D, Testa AC, Bourne T, Ferrazzi E, Ameye L, Konstantinovic ML, et al. Logistic regression model to distinguish between the benign and malignant adnexal mass before surgery: a multicenter study by the International Ovarian Tumor Analysis Group. J Clin Oncol. 2005;23(34):8794-801.

15. Sladkevicius $P$, Valentin L. Intra- and interobserver agreement when describing adnexal masses using the International Ovarian Tumor Analysis terms and definitions: a study on three-dimensional ultrasound volumes. Ultrasound Obstet Gynecol. 2013;41(3):318-27.

16. Arezzo F, Franchi D, Loizzi V, Cataldo V, Lombardi C, Cazzato G, et al. Blue mass in the pelvis: serous cystadenofibroma of the peritoneum. Ultrasound Obstet Gynecol. 2021.

17. Levine D, Brown DL, Andreotti RF, Benacerraf B, Benson CB, Brewster WR, et al. Management of asymptomatic ovarian and other adnexal cysts imaged at US: Society of Radiologists in Ultrasound Consensus Conference Statement. Radiology. 2010;256(3):943-54. 
18. Levine D, Brown DL, Andreotti RF, Benacerraf B, Benson CB, Brewster WR, et al. Management of asymptomatic ovarian and other adnexal cysts imaged at US Society of Radiologists in Ultrasound consensus conference statement. Ultrasound Q. 2010;26(3):121-31.

19. Amor F, Vaccaro H, Alcazar JL, Leon M, Craig JM, Martinez J. Gynecologic imaging reporting and data system: a new proposal for classifying adnexal masses on the basis of sonographic findings. $J$ Ultrasound Med. 2009;28(3):285-91.

20. Arezzo F, Loizzi V, La Forgia D, Moschetta M, Tagliafico AS, Cataldo V, et al. Radiomics Analysis in Ovarian Cancer: A Narrative Review. Applied Sciences. 2021;11(17):7833.

21. Andreotti RF, Timmerman D, Strachowski LM, Froyman W, Benacerraf BR, Bennett GL, et al. O-RADS US Risk Stratification and Management System: A Consensus Guideline from the ACR OvarianAdnexal Reporting and Data System Committee. Radiology. 2020;294(1):168-85.

22. Venerito V, Angelini O, Cazzato G, Lopalco G, Maiorano E, Cimmino A, et al. A convolutional neural network with transfer learning for automatic discrimination between low and high-grade synovitis: a pilot study. Intern Emerg Med. 2021.

23. Venerito V, Angelini O, Fornaro M, Cacciapaglia F, Lopalco G, lannone F. A Machine Learning Approach for Predicting Sustained Remission in Rheumatoid Arthritis Patients on Biologic Agents. JCR: Journal of Clinical Rheumatology. 2021;Publish Ahead of Print.

24. Cazzato G, Colagrande A, Cimmino A, Arezzo F, Loizzi V, Caporusso C, et al. Artificial Intelligence in Dermatopathology: New Insights and Perspectives. Dermatopathology (Basel). 2021;8(3):418-25.

25. Johnson KW, Torres Soto J, Glicksberg BS, Shameer K, Miotto R, Ali M, et al. Artificial Intelligence in Cardiology. J Am Coll Cardiol. 2018;71(23):2668-79.

26. Arezzo F, La Forgia D, Venerito V, Moschetta M, Tagliafico AS, Lombardi C, et al. A Machine Learning Tool to Predict the Response to Neoadjuvant Chemotherapy in Patients with Locally Advanced Cervical Cancer. Applied Sciences. 2021;11(2):823.

27. Pandit A, Radstake T. Machine learning in rheumatology approaches the clinic. Nat Rev Rheumatol. 2020;16(2):69-70.

28. Baldini C, Ferro F, Luciano N, Bombardieri S, Grossi E. Artificial neural networks help to identify disease subsets and to predict lymphoma in primary Sjogren's syndrome. Clin Exp Rheumatol. 2018;36 Suppl 112(3):137-44.

29. Cohen JF, Korevaar DA, Altman DG, Bruns DE, Gatsonis CA, Hooft L, et al. STARD 2015 guidelines for reporting diagnostic accuracy studies: explanation and elaboration. BMJ Open. 2016;6(11):e012799.

30. Collins GS, Reitsma JB, Altman DG, Moons KG. Transparent reporting of a multivariable prediction model for individual prognosis or diagnosis (TRIPOD): the TRIPOD statement. BJOG. 2015;122(3):434-43.

31. Chen T, Guestrin C. XGBoost: A Scalable Tree Boosting System. Proceedings of the 22nd ACM SIGKDD International Conference on Knowledge Discovery and Data Mining; San Francisco, California, USA: Association for Computing Machinery; 2016. p. 785-94. 
32. Casalino G, Vessio G, Consiglio A, editors. Evaluation of Cognitive Impairment in Pediatric Multiple Sclerosis with Machine Learning: An Exploratory Study of miRNA Expressions. 2020 IEEE Conference on Evolving and Adaptive Intelligent Systems (EAIS); 2020 27-29 May 2020.

33. Kamel E, Sheikh S, Huang X. Data-driven predictive models for residential building energy use based on the segregation of heating and cooling days. Energy. 2020;206:118045.

34. Zeng X, Chen Y, Tao C, Alphen Dv, editors. Feature Selection Using Recursive Feature Elimination for Handwritten Digit Recognition. 2009 Fifth International Conference on Intelligent Information Hiding and Multimedia Signal Processing; 2009 12-14 Sept. 2009.

35. LeCun Y, Bengio Y, Hinton G. Deep learning. Nature. 2015;521(7553):436-44.

36. Buitinck L, Louppe G, Blondel M, Pedregosa F, Mueller A, Grisel O, et al. API design for machine learning software: experiences from the scikit-learn project2013 September 01, 2013: [arXiv:1309.0238 p.]. Available from: https://ui.adsabs.harvard.edu/abs/2013arXiv1309.0238B.

37. Altukhova 0 . Choice of method imputation missing values for obstetrics clinical data. Procedia Computer Science. 2020;176:976-84.

38. Xiao M, Yan C, Fu B, Yang S, Zhu S, Yang D, et al. Risk prediction for postpartum depression based on random forest. Zhong Nan Da Xue Xue Bao Yi Xue Ban. 2020;45(10):1215-22.

39. Rawashdeh H, Awawdeh S, Shannag F, Henawi E, Faris H, Obeid N, et al. Intelligent system based on data mining techniques for prediction of preterm birth for women with cervical cerclage. Comput Biol Chem. 2020;85:107233.

40. Zhang $H$, Wang $X$, Ding $R$, Shen $L, G a o ~ P, X u ~ H$, et al. Characterization and imaging of surgical specimens of invasive breast cancer and normal breast tissues with the application of Raman spectral mapping: A feasibility study and comparison with randomized single-point detection method. Oncol Lett. 2020;20(3):2969-76.

41. Khalilia M, Chakraborty S, Popescu M. Predicting disease risks from highly imbalanced data using random forest. BMC Medical Informatics and Decision Making. 2011;11(1):51.

42. Krstajic D, Buturovic LJ, Leahy DE, Thomas $S$. Cross-validation pitfalls when selecting and assessing regression and classification models. J Cheminform. 2014;6(1):10.

43. Berrar D. Performance Measures for Binary Classification. In: Ranganathan S, Gribskov M, Nakai K, Schönbach C, editors. Encyclopedia of Bioinformatics and Computational Biology. Oxford: Academic Press; 2019. p. 546-60.

44. Kuhn M, Johnson K. Applied Predictive Modeling2013.

45. Matsuo K, Purushotham S, Jiang B, Mandelbaum RS, Takiuchi T, Liu Y, et al. Survival outcome prediction in cervical cancer: Cox models vs deep-learning model. Am J Obstet Gynecol. 2019;220(4):381 e1- e14.

46. Tailor A, Jurkovic D, Bourne TH, Collins WP, Campbell S. Sonographic prediction of malignancy in adnexal masses using an artificial neural network. Br J Obstet Gynaecol. 1999;106(1):21-30. 
47. Biagiotti R, Desii C, Vanzi E, Gacci G. Predicting ovarian malignancy: application of artificial neural networks to transvaginal and color Doppler flow US. Radiology. 1999;210(2):399-403.

48. Khazendar S, Sayasneh A, Al-Assam H, Du H, Kaijser J, Ferrara L, et al. Automated characterisation of ultrasound images of ovarian tumours: the diagnostic accuracy of a support vector machine and image processing with a local binary pattern operator. Facts Views Vis Obgyn. 2015;7(1):7-15.

49. Chiappa V, Interlenghi M, Bogani G, Salvatore C, Bertolina F, Sarpietro G, et al. A decision support system based on radiomics and machine learning to predict the risk of malignancy of ovarian masses from transvaginal ultrasonography and serum CA-125. Eur Radiol Exp. 2021;5(1):28.

50. Martinez-Mas J, Bueno-Crespo A, Khazendar S, Remezal-Solano M, Martinez-Cendan JP, Jassim S, et al. Evaluation of machine learning methods with Fourier Transform features for classifying ovarian tumors based on ultrasound images. PLoS One. 2019;14(7):e0219388.

51. Hwangbo S, Kim SI, Kim JH, Eoh KJ, Lee C, Kim YT, et al. Development of Machine Learning Models to Predict Platinum Sensitivity of High-Grade Serous Ovarian Carcinoma. Cancers (Basel). 2021;13(8).

52. Shannon NB, Tan LLY, Tan QX, Tan JW, Hendrikson J, Ng WH, et al. A machine learning approach to identify predictive molecular markers for cisplatin chemosensitivity following surgical resection in ovarian cancer. Sci Rep. 2021;11(1):16829.

\section{Supplementary}

The Supplementary Materials are not available with this version

\section{Figures}




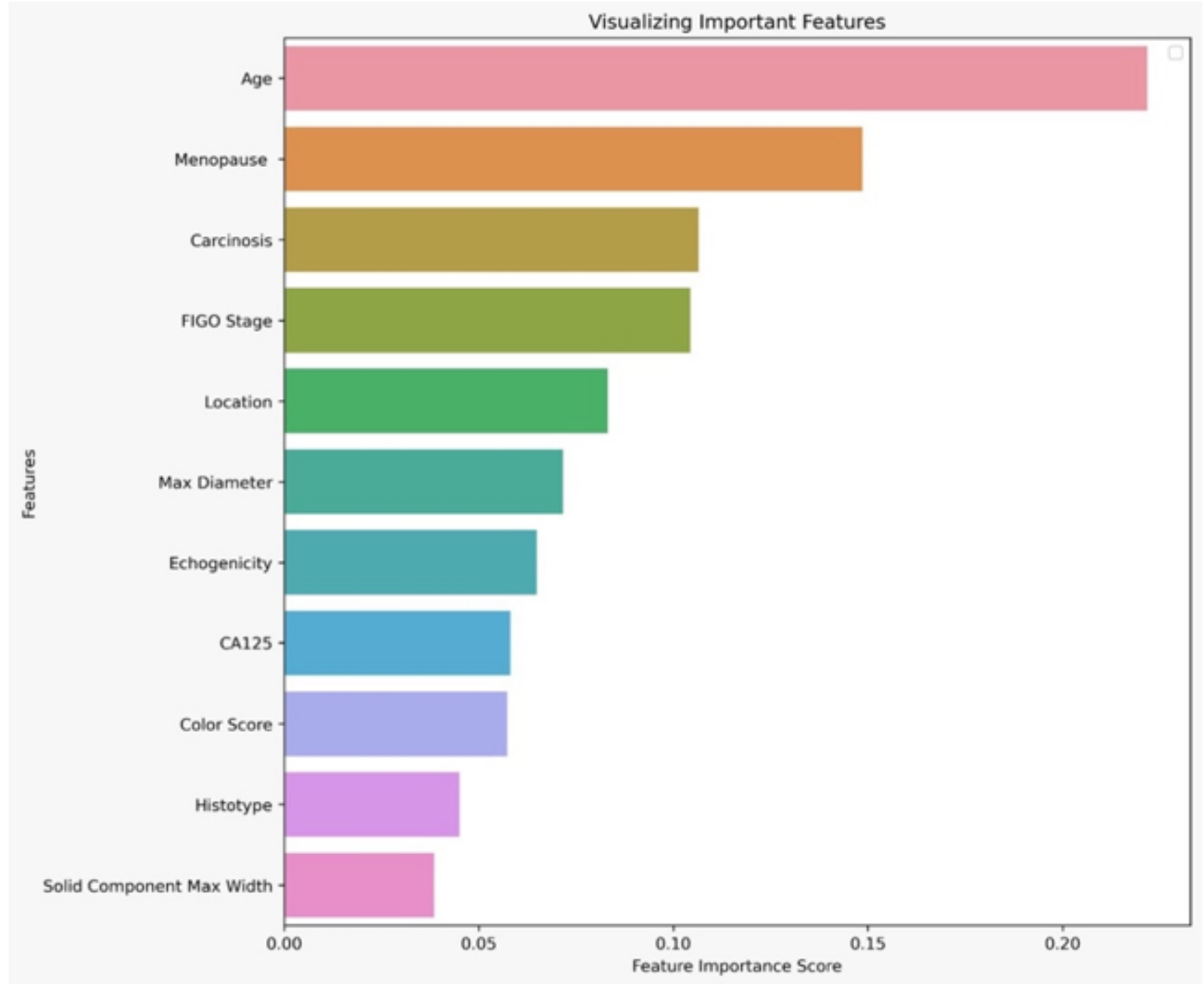

Figure 1

Feature importance of the attribute coreset

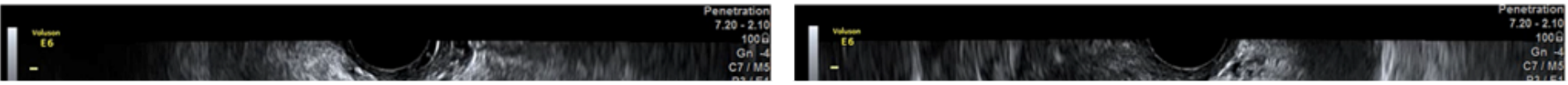




\section{Figure 2}

Size lesions measurement

The size of the lesion are measured as the largest three diameters (in $\mathrm{mm}$ ) in two perpendicular planes. The largest diameter was found to be one of the most important features to predict PFS
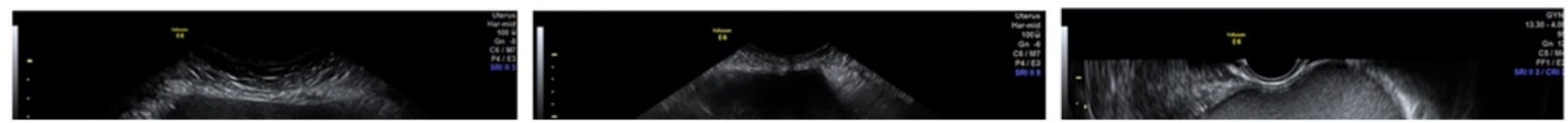

\section{Figure 3}

\section{Echogenicity of cyst fluid}

The echogenicity of cyst fluid in tumors not classified as solid is described as anechoic (Panel a, low level (homogeneous low level echogenic) (Panel b) or ground glass (homogeneously dispersed echogenic cystic contents) (Panel c). 

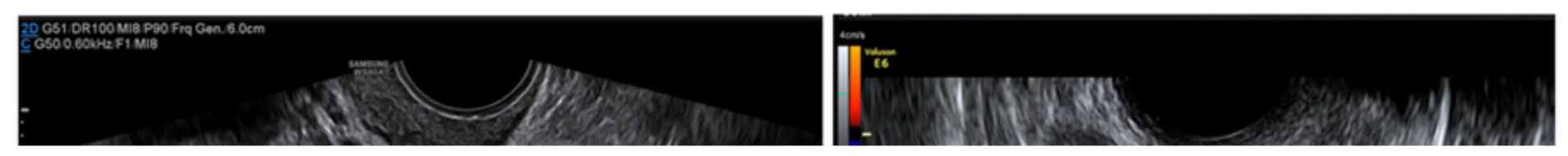

\section{Figure 4}

Assessment of blood flow

The assessment of blood flow is a subjective assessment evaluated with a color scale.

Panel a. Color score 1: no flow

Panel b. Color score 2: minimal flow

Panel c. Color score 3: moderate flow

Panel d. Color score 4: intense flow

The color score evaluation was found to be one of the most important features to predict PFS. 


\section{Figure 5}

Major solid component diameter measurement

The largest solid component in a cystic-solid tumors is measures separately with the assessment of two or three diameters in two perpendicular planes.

The largest solid component was found to be one of the most important features to predict PFS.

\section{Figure 6}

Ultrasound finding of carcinosis

Ultrasound assessment of carcinosis was found to be one of the most important features to predict PFS. 


\section{Figure 7}

Receiver operating characteristics curve for Random Forest (box (A)), Logistic Regression (box (B)) and Knearest neighbors (box (C)) models.

\section{Figure 8}

Calibration diagnostics for RFF model. 12-month PFS roughly happened with an observed relative frequency consistent with the forecast value, showing good calibration. 